\title{
A visão dos docentes do curso de medicina de uma instituição de ensino superior de
}

\section{Palmas - Tocantins sobre a telemedicina}

\author{
The vision of medical school teachers of a higher education institution in Palmas - Tocantins on
} telemedicine

\section{La opinión de los profesores del curso de medicina de una institución de educación superior en Palmas - Tocantins sobre telemedicine}

\section{Resumo}

A telemedicina é um instrumento que utiliza a tecnologia da comunicação e possibilita fornecer acesso à saúde, solucionando problemas nos quais a distância seria um fator crítico. Objetivo: Conhecer a visão dos docentes do curso de medicina de uma Instituição de Ensino superior em Palmas - Tocantins sobre a telemedicina. Metodologia: pesquisa transversal e descritiva, com abordagem quantitativa e qualitativa, desenvolvida com professores do curso de medicina do $1^{\circ}$ ao $8^{\circ}$ período do Instituto Tocantinense Presidente Antônio Carlos - ITPAC Palmas. Os dados foram coletados no período de maio à setembro, através de um questionário online disponibilizado por meio do GoogleForms ${ }^{\circledR}$ com o intuito de analisar a visão dos docentes da IES diante do crescimento e difusão da telemedicina, contendo 21 questões que abordavam sobre essa temática. Resultados: 34,6\% da amostra desacreditam na garantia integral da segurança/sigilo ao paciente durante os atendimentos remotos, e que as consultas utilizando a telemedicina não substituem o acompanhamento presencial, pois muitas especialidades médicas carecem da relação presencial médico-paciente para obter sucesso na assistencia à saúde . Ressalta-se que os pontos positivos também foram idenficiados, como: maior alcance à população, possibilidade do contato entre especialistas e o alívio na sobrecarga do Sistema Único de Saúde. Conclusão: A visão dos docentes no que se refere ao uso da telemedicina é multifacetária. Possui pontos negativos como a dificuldade de atender algumas especialidades, e pontos positivos como: ampliação do acesso e compartilhamento de saberes entre os profissionais. Sugere-se a ampliação dos estudos e detabates envolvendo o tema.

Palavras-chave: Telemedicina; Telessaúde; Docentes; Ensino; Informações; Discussão.

\begin{abstract}
Telemedicine is an instrument that uses communication technology and makes it possible to provide access to health, solving problems in which distance would be a critical factor. Objective: To know the view of the professors of the medical course of a higher education institution in Palmas - Tocantins about telemedicine. Methodology: cross-
\end{abstract}


sectional and descriptive research, with a quantitative and qualitative approach, developed with medical school professors from the 1st to the 8th period of Instituto Tocantinense Presidente Antônio Carlos - ITPAC Palmas. Data were collected from May to September, through an online questionnaire made available through GoogleForms ${ }^{\circledR}$ in order to analyze the view of the HEI professors on the growth and diffusion of telemedicine, containing 21 questions that addressed this theme. Results: $34.6 \%$ of the sample disbelieve in the full guarantee of safety/secrecy to the patient during remote care, and that consultations using telemedicine do not replace face-to-face follow-up, as many medical specialties lack the face-to-face doctor-patient relationship to achieve success in health care. It is noteworthy that the positive points were also identified, such as: greater reach to the population, possibility of contact between specialists and relief from the burden of the Unified Health System. Conclusion: The view of the professors regarding the use of telemedicine is multifaceted. It has negative points such as the difficulty of attending some specialties, and positive points such as: expanding access and sharing of knowledge among professionals. The expansion of studies and debates involving the subject is suggested.

Keywords: Telemedicine; Telehealth; Teachers; Teaching; Information; Discussion.

\section{Resumen}

La telemedicina es un instrumento que utiliza la tecnología de la comunicación y permite brindar acceso a la salud, resolviendo problemas en los que la distancia sería un factor crítico. Objetivo: Conocer la visión de los profesores del curso de medicina de una institución de educación superior en Palmas - Tocantins sobre la telemedicina. Metodología: investigación transversal y descriptiva, con enfoque cuantitativo y cualitativo, desarrollada con profesores de las facultades de medicina del $1^{\circ}$ al $8^{\circ}$ período del Instituto Tocantinense Presidente Antônio Carlos - ITPAC Palmas. Los datos fueron recolectados de mayo a septiembre, a través de un cuestionario en línea disponible a través de GoogleForms ${ }^{\circledR}$ con el fin de analizar la visión de los profesores de IES sobre el crecimiento y difusión de la telemedicina, conteniendo 21 preguntas que abordaron este tema. Resultados: El 34,6\% de la muestra no cree en la total garantía de seguridad / secreto al paciente durante la atención remota, y que las consultas mediante telemedicina no sustituyen al seguimiento presencial, ya que muchas especialidades médicas carecen del presencial. enfrentar la relación médico-paciente para lograr el éxito en la atención médica. Cabe mencionar que también se identificaron los puntos positivos, tales como: mayor alcance a la población, posibilidad de contacto entre especialistas y alivio de la carga del Sistema Único de Salud. Conclusión: La visión de los profesores sobre el uso de la telemedicina es multifacética. Tiene puntos negativos como la dificultad para atender algunas especialidades, y puntos positivos como: ampliación del acceso e intercambio de conocimientos entre los profesionales. Se sugiere la ampliación de estudios y debates relacionados con el tema.

Palabras clave: Telemedicina; Telesalud; Profesores; Enseñando; Información; Discusión.

\section{Introdução}

As lacunas da universalização da saúde que precisavam serem preenchidas desencadearam a necessidade de um novo modelo de atendimento, o qual busca trazer facilidades para toda a rede hospitalar e garantir o acesso a uma parte da população que não é assistida. Assim, juntamente com a ascensão da tecnologia, houve, no século XX, o surgimento da telemedicina que por meio das tecnologias da informação e comunicação visa garantir a integralidade da atenção à saúde com um acesso eficiente, de qualidade e resolutivo.

Segundo essa perspectiva, alguns acontecimentos vêm criando condições favoráveis para um avanço cada vez maior da telessaúde, como o controle dos gastos públicos e privados com a saúde em um cenário mundial, o aumento das doenças agudas e crônicas que necessitam de um acompanhamento multidisciplinar que explicitou a importância do manejo do atendimento remoto.

Dessa forma, esse artigo apresenta a perspectiva dos docentes da faculdade ITPAC-Palmas acerca da telessaúde, levando em consideração a importância da temática para construção de um cenário médico de maior assistência.

\section{Metodologia}

Trata-se de estudo transversal e descritivo, com abordagem quantitativa e qualitativa. Ressalta-se que os "métodos qualitativos e quantitativos não se excluem e podem ser importantes se complementando e permitindo um melhor entendimento dos fenômenos em estudo" (Pereira, Shitsuka, Parreira \& Shitsuka, 2018, p. 69). A pesquisa foi desenvolvida no período de dezembro de 2020 até abril de 2021, com professores do $1^{\circ}$ ao $8^{\circ}$ período do curso de medicina do Instituto 
Tocantinense Presidente Antônio Carlos - ITPAC Palmas.

A pesquisa foi realizada seguindo todas as exigências do Conselho Nacional de Saúde que rege a Resolução 466/12, que normatiza pesquisa envolvendo seres humanos, enviado no dia 10 de março de 2021 ao Comitê de Ética UNITPAC Araguaína, sendo aprovado no dia 23 de março de 2021.

Os critérios de inclusão foram: 1. Médicos docentes do curso de Medicina da ITPAC- Palmas que lecionam do $1^{\circ}$ ao $8^{\circ}$ período; 2 . Contato prévio com a Telemedicina; 3 . Aceite participar da pesquisa e assine o Termo de Consentimento Livre e Esclarecido - TCLE. Foram excluídos os docentes que não prestam serviços no ITPAC-Palmas, que não são vinculados ao curso de medicina, que não são médicos, que possuem relação com empresas de telemedicina, que nunca tiveram contato com a telemedicina e que não assinaram o TCLE.

Após o levantamento e seleção foram identificados 32 docentes, sendo que 6 (18,75\%) foram descartados pelos critérios de exclusão, restando 26 (81,25\%) que atenderam aos critérios da pesquisa. O questionário foi elaborado e adequado para ferramenta GoogleForms ${ }^{\circledR}$, permitindo a resposta instantânea e arquivamento para posterior análise dos dados. Para aplicação do instrumento e aceite através da assinatura do TLCE foi utilizado o aplicativo de mensagem WhatsApp®.

Os dados foram coletados no período de maio à setembro de 2021. O questionário continha 21 perguntas de múltipla escolha e uma dissertativa, seguindo os critérios de inclusão e exclusão. As perguntas objetivavam entender a visão dos docentes sobre a telemedicina e seus avanços, e as alternativas se dividiram entre 14 questões utilizando a escala de Likert visando validar com clareza as informações colhidas, nos quais foram graduadas em 5 graus diferentes: 1- concordo totalmente, 2- concordo parcialmente, 3- discordo parcialmente, 4- discordo totalmente, 5- não concordo e nem discordo, e as outras 5 questões foram alteradas de acordo com a solicitação dos respondentes para facilitar o entendimento do questionário.

\section{Resultados e Discussão}

Foi realizada uma análise descritiva dos dados, nos quais os resultados são apresentados em valores absolutos e percentuais a partir dos dados arquivados na plataforma GoogleForms ${ }^{\circledR}$. Essa ferramenta oferece recursos para observação dos dados estruturados, por meio de números e gráficos automaticamente fornecidos.

O questionário contou com 32 respondentes, nos quais $6(18,75 \%)$ foram descartados pelos critérios de exclusão, restando $26(81,25 \%)$ que se adequaram à pesquisa.

Dos professores que responderam o questionário, 26 (100\%) são médicos, já ouviram falar sobre a telemedicina e nunca trabalharam ou trabalham nessa área, segundo análise das perguntas 2, 3, 4 e 11. Dentro da população estudada, 9 $(34,6 \%)$ nunca trabalharam ou tiveram interesse em trabalhar com a telemedicina, enquanto 17 (65,4\%) delas já demonstraram interesse em atuar nesse meio, de acordo com análise da pergunta 5.

De acordo com Oliveira, Tokarski, Japiassu, \& Silva (2020), a telemedicina começou a entrar em evidência no Brasil ao fim da década de 1990, tendo sido difundida de forma gradual ao longo dos próximos anos. Inicialmente, foi instituído o Programa Nacional Telessaúde Brasil em Redes por meio da Portaria do Ministério da Saúde no 35 de janeiro de 2007 que possibilita uma educação permanente em saúde (EPS) e apoio assistencial por meio de ferramentas e tecnologias da informação e educação para profissionais do SUS, garantindo, assim, a melhoria da qualidade no atendimento. Atualmente, devido à situação pandêmica na qual estamos inseridos, a telemedicina ganha cada vez mais força e alcança diferentes ramos da saúde.

No Brasil a telemedicina pode ser exercida em 3 moldes principais: Teleorientação, Telemonitoramento e Teleinterconsulta. A primeira possibilita aos médicos realizarem orientações e encaminhamentos, semelhante a triagem, em pacientes que estejam em suas residências. O Telemonitoramento permite o monitoramento remoto dos parâmetros de saúde e/ou doença, sendo estes supervisionados ou orientados por médicos. Já a Teleinterconsulta 
permite a troca exclusiva de informações e opiniões entre médicos, sejam estas para auxílio diagnóstico e/ou terapêutico. (Brito \& Leitão, 2020, p.5)

Segundo Khouri (2003) a história da telemedicina é importante e não pode ser isolada da de outros ramos de progresso da humanidade. A telemedicina se mostra bastante promissora e inovadora, viabilizando os serviços de saúde principalmente quando a distância é um fator crítico, tendo em vista a grande demanda e a dificuldade ao acesso a algumas áreas geograficamente isoladas, esse meio de comunicação faz-se necessário para um maior alcance de pessoas (Machado, Carvalho, Mataresi, Mendonça, Cardoso, Yogi, Rigato, Salazar, 2010). Além disso, o atendimento remoto proporciona a supressão do deslocamento do paciente, otimiza o tempo dos profissionais e reduz os custos operacionais que podem ser utilizados em outras melhorias para a saúde pública. No Brasil, o estado da Amazônia apresenta-se como um polo regional, principalmente pela carência de profissionais, meios diagnósticos e serviços de referência (Rezende et al., 2010). Nota-se que 61,5\% entrevistados concordam parcialmente que a telemedicina é uma alternativa para aliviar a sobrecarga do SUS, já 30,7\% acreditam não ser possível reduzir a sobrecarga do Sistema Único de Saúde do Brasil - SUS, conforme os dados apresentados na Tabela 1, isso devido as seguintes limitações: a inviabilidade de realizar o exame físico, ausência de internet em alguns locais e a falta de tecnologias para manter a segurança dos prontuários.

Tabela 1. Análise das perguntas 6 e 13 do questionário aplicado aos docentes.

\begin{tabular}{|c|c|c|c|c|c|c|}
\hline $\begin{array}{l}\text { Número da } \\
\text { questão }\end{array}$ & Pergunta & $\begin{array}{c}\text { Grau 1- Concordo } \\
\text { Totalmente }\end{array}$ & $\begin{array}{l}\text { Grau 2- Concordo } \\
\text { Parcialmente }\end{array}$ & $\begin{array}{l}\text { Grau 3- Discordo } \\
\text { Parcialmente }\end{array}$ & $\begin{array}{c}\text { Grau 4- } \\
\text { Discordo } \\
\text { Totalmente }\end{array}$ & $\begin{array}{c}\text { Grau 5- Nem } \\
\text { concordo e } \\
\text { nem discordo }\end{array}$ \\
\hline 6 & $\begin{array}{c}\text { Você vê a } \\
\text { Telemedicina como } \\
\text { uma alternativa de } \\
\text { aliviar a sobrecarga } \\
\text { do SUS? }\end{array}$ & 2 & 16 & 5 & 3 & 0 \\
\hline 13 & $\begin{array}{c}\text { É possível afirmar } \\
\text { que a telemedicina } \\
\text { será eficaz para } \\
\text { solucionar a } \\
\text { demanda do } \\
\text { paciente? }\end{array}$ & 1 & 14 & 6 & 5 & 0 \\
\hline
\end{tabular}

Fonte: Autores (2021).

É importante ressaltar que a telemedicina ainda é vista por muitos profissionais com desconfiança, mas que segundo Mariani e Pêgo-Fernandes (2012, p.1) “seu crescimento é inequívoco e sustentado. Iniciativas e programas baseados em telemedicina são cada vez mais presentes na prática moderna da medicina”.

É sabido que durante uma consulta online não há um contato médico-paciente sólido, já que através das telas alguns aspectos da consulta médica ficam prejudicados. E, a partir da análise dos entrevistados percebe-se que essa ideia se faz fortemente presente entre a comunidade dos médicos docentes. Rezende et. al (2010, p.1) aponta que "a forma convencional de atendimento na área de saúde é representada pelo encontro presencial. Atualmente, os recursos tecnológicos conseguem promover um "encontro virtual" entre os profissionais de saúde e o paciente, ou entre outros profissionais, sempre que a distância ou outros fatores impedirem o encontro pessoal. Essa característica peculiar foge aos padrões habituais convencionais, muda paradigmas, e tem implicações éticas e legais". Com base na Tabela 2 nota-se que um número expressivo, 73,1\% dos entrevistados, concordam plenamente que a telemedicina possui limitações, e 46,2\% concordam parcialmente que prejudica a relação médico-paciente. 
Tabela 2. Análise das perguntas 7 e 8 do questionário aplicado aos docentes.

\begin{tabular}{|c|c|c|c|c|c|c|}
\hline $\begin{array}{l}\text { Número da } \\
\text { questão }\end{array}$ & Pergunta & $\begin{array}{c}\text { Grau 1- } \\
\text { Concordo } \\
\text { Totalmente }\end{array}$ & $\begin{array}{l}\text { Grau 2- Concordo } \\
\text { Parcialmente }\end{array}$ & $\begin{array}{l}\text { Grau 3- Discordo } \\
\text { Parcialmente }\end{array}$ & $\begin{array}{c}\text { Grau 4- } \\
\text { Discordo } \\
\text { Totalmente }\end{array}$ & $\begin{array}{c}\text { Grau 5- Nem } \\
\text { concordo e nem } \\
\text { discordo }\end{array}$ \\
\hline 7 & $\begin{array}{c}\text { Você acha que a } \\
\text { Telemedicina possui } \\
\text { limitações? }\end{array}$ & 19 & 7 & 0 & 0 & 0 \\
\hline 8 & $\begin{array}{l}\text { Você considera que o } \\
\text { atendimento remoto } \\
\text { prejudica a relação } \\
\text { médico- paciente? }\end{array}$ & 6 & 12 & 5 & 3 & 0 \\
\hline
\end{tabular}

Fonte: Autores (2021).

Entretanto, ainda que tenhamos limitações dentro dos atendimentos virtuais, as diversas modalidades de consulta, como: a teleorientação, o telemonitoramento e a teleinterconsulta, conferem uma maior diversidade de atuação dos profissionais no âmbito dos serviços online, e, considerando a crescente adesão da população ao mundo digital, o meio médico tem necessitado adaptar-se às novas circunstâncias. Com isso, é possível agilizar a dinâmica do serviço de saúde através do contato rápido entre especialistas que antes não seria viável, ofertar atendimentos especializados, alcançar áreas isoladas, e, assim, assegurar uma maior qualidade no atendimento ao paciente. Os dados apresentados na Tabela 3 mostram que $61,5 \%$ acreditam que o atendimento pode ser seguro e $61,5 \%$ creem que é possível manter a ética.

Tabela 3. Análise das perguntas 9, 14, 16, 17 e 19 do questionário aplicado aos docentes.

\begin{tabular}{|c|c|c|c|c|c|c|}
\hline $\begin{array}{l}\text { Número da } \\
\text { questão }\end{array}$ & Pergunta & $\begin{array}{l}\text { Grau 1- } \\
\text { Concordo } \\
\text { Totalmente }\end{array}$ & $\begin{array}{l}\text { Grau 2- Concordo } \\
\text { Parcialmente }\end{array}$ & $\begin{array}{l}\text { Grau 3- Discordo } \\
\text { Parcialmente }\end{array}$ & $\begin{array}{c}\text { Grau 4- } \\
\text { Discordo } \\
\text { Totalmente }\end{array}$ & $\begin{array}{c}\text { Grau 5- Nem } \\
\text { concordo e } \\
\text { nem discordo }\end{array}$ \\
\hline 9 & $\begin{array}{l}\text { Você acredita que o } \\
\text { atendimento remoto pode } \\
\text { ser seguro para o exercício } \\
\text { da Medicina? }\end{array}$ & 1 & 16 & 6 & 3 & 0 \\
\hline 14 & $\begin{array}{l}\text { É possível manter a } \\
\text { qualidade e a ética no } \\
\text { atendimento remoto? }\end{array}$ & 4 & 16 & 3 & 3 & 0 \\
\hline 16 & $\begin{array}{l}\text { Você conhece as leis que } \\
\text { regulamentam a } \\
\text { Telemedicina hoje no } \\
\text { Brasil? }\end{array}$ & 3 & 17 & 0 & 6 & 0 \\
\hline 17 & $\begin{array}{c}\text { Você concorda com as leis } \\
\text { vigentes do Brasil sobre a } \\
\text { Telemedicina? }\end{array}$ & 1 & 11 & 4 & 1 & 9 \\
\hline 19 & $\begin{array}{l}\text { Você possui algum } \\
\text { conhecimento acerca das } \\
\text { redes de serviços que } \\
\text { oferecem o atendimento } \\
\text { por Telemedicina no } \\
\text { estado do Tocantins? }\end{array}$ & 2 & 10 & 4 & 6 & 4 \\
\hline
\end{tabular}

Fonte: Autores (2021).

Segundo Avila (2003), a relação médico-paciente nos moldes tradicionais não pode ser eliminada ou substituída de imediato, pois alteraria alguns princípios tradicionais que regulam o exercício ético da Medicina. Assim, é essencial que o 
atendimento online seja moldado no respeito ao sigilo, na confidencialidade e na privacidade das informações fornecidas pelos pacientes, e sua evolução do presencial para o digital aconteça de forma gradativa e respeitando os limites do exercício profissional. Dito isso, o Conselho Federal de Medicina estabelece algumas regras a respeito da ética médica que garantem a segurança dos envolvidos.

As regras correntes do consentimento e confidencialidade do paciente também se aplicam às situações da Telemedicina. A informação sobre o paciente só pode ser transmitida ao médico ou a outro profissional de saúde se isso for permitido pelo paciente com seu consentimento esclarecido. A informação transmitida deve ser pertinente ao problema em questão. Devido aos riscos de filtração de informações inerentes a certos tipos de comunicação eletrônica, o médico tem a obrigação de assegurar que sejam aplicadas todas as normas de medidas de segurança estabelecidas para proteger a confidencialidade do paciente. Qualidade da atenção e segurança na Telemedicina (França, 2009, p. 17).

É importante que as leis que regem os atendimentos remotos sejam claras para todos os envolvidos nesse processo, a fim de manter os aspectos éticos da profissão médica. Pensando nisso, o CFM aprovou diversas resoluções, dentre elas a de $\mathrm{n}^{\circ}$ 1639, que elucida as normas técnicas para manuseio e armazenamento dos prontuários médicos, de modo que sejam feitas de forma segura. Para isso, é importante que sejam seguidos alguns critérios como o acesso restrito e limitado aos usuários e profissionais, por meio de senhas exclusivas e métodos que sejam capazes autenticarem os perfis cadastrados, tornando a entrada única e individualizada. Além disso, existem outras resoluções dispostas que asseguram a integridade do paciente, como a nº $1643 / 2002$ que tem por finalidade garantir que os dados cadastrados só devam ser transmitidos para outro profissional médico mediante aprovação do paciente, o que evita a distribuição dos dados colhidos dentro das consultas, e, assim, garante segurança e ética nos atendimentos de telemedicina.

A orientação ética para o exercício da medicina advém do Código de Ética Médica (CEM), promulgado pela resolução $n^{\circ} 1246$ (de 8 de janeiro de 1988) do Conselho Federal de Medicina (CFM). Esse código contém alguns artigos relacionados ao exercício da telemedicina (12). Os principais são: • Artigo 62: É vedado ao médico prescrever tratamento ou outros procedimentos sem exame direto do paciente, salvo em casos de urgência e impossibilidade comprovada de realizá-lo, devendo, nesse caso, fazê-lo imediatamente cessado o impedimento. • Artigo 102: É vedado ao médico revelar informações sobre os pacientes sem o consentimento dos mesmos, exceto em situações que possam se configurar como dever legal ou justa causa. A proibição permanece em situações que chegam a juízo, bem como nas solicitações das seguradoras. - Artigo 104: É vedado ao médico fazer referência a casos clínicos identificáveis, exibir pacientes ou seus retratos em anúncios profissionais ou na divulgação de assuntos médicos. • Artigo 108: É vedado ao médico facilitar o manuseio e conhecimento dos prontuários, papeletas e demais folhas de observações médicas sujeitas ao segredo profissional, por pessoas não obrigadas ao mesmo compromisso. • Artigo 134: É vedado ao médico dar consulta, diagnóstico ou prescrição por intermédio de qualquer veículo de comunicação de massa. (Rezende et al., 2010, p.2)

Em outubro de 1999, a 51ª Assembleia Geral da Associação Médica Mundial, realizada em Tel Aviv, Israel, adotou a "Declaração de Tel Aviv sobre responsabilidades e normas éticas na utilização da telemedicina". Essa declaração foi adotada como modelo de orientação ética para as diversas atividades de telemedicina. Como postulado básico, a declaração ressalta que “independente do sistema de telemedicina que o médico utiliza os princípios da ética médica, a que está sujeita mundialmente a profissão médica, nunca devem ser comprometidos” (Rezende et al., 2010).

Ao analisar os dados apresentados na tabela 4 , nota-se que $57,7 \%$ concordam parcialmente que a Telemedicina pode trazer acessibilidade à saúde, conectando diferentes áreas geográficas que antes não possuíam acesso a serviços qualificados, possibilitando melhor assistência ao paciente devido à expansão nas redes de networking. Esse processo culminou com a aproximação dos médicos generalistas com os médicos especialistas, que antes ocasionavam elevados custos tanto ao paciente, quanto ao sistema gestor. 
Tabela 4. Análise das perguntas 10 e 12 do questionário aplicado aos docentes.

\begin{tabular}{|c|c|c|c|c|c|c|}
\hline $\begin{array}{l}\text { Número da } \\
\text { questão }\end{array}$ & Pergunta & $\begin{array}{l}\text { Grau 1- } \\
\text { Concordo } \\
\text { Totalmente }\end{array}$ & $\begin{array}{c}\text { Grau 2- } \\
\text { Concordo } \\
\text { Parcialmente }\end{array}$ & $\begin{array}{l}\text { Grau 3- Discordo } \\
\text { Parcialmente }\end{array}$ & $\begin{array}{c}\text { Grau 4- } \\
\text { Discordo } \\
\text { Totalmente }\end{array}$ & $\begin{array}{c}\text { Grau 5- Nem } \\
\text { concordo e nem } \\
\text { discordo }\end{array}$ \\
\hline
\end{tabular}

É possível afirmar que a

10

Telemedicina trouxe uma democratização do acesso à saúde?

Você acredita que a

12 telemedicina pode reduzir custos do serviço de saúde?

Você concorda que a

15 Telemedicina possa ser acessível a todos?
2

15

5

3

1

6

14

3

3

0

1

13

5

7

Fonte: Autores (2021).

As tecnologias de informação e comunicação são capazes de transformar a organização e a prestação da assistência médica de diversas formas. Quando bem aplicada, pode trazer bons resultados econômicos, e, assim, estimular a realocação de recursos públicos em novas tecnologias e infraestrutura dos locais de atendimento (Resende et al., 2019). Dessa forma, todos se beneficiariam, logo que, seria possível aumentar a disponibilidade de consultas e elevar a agilidade nos atendimentos, que a médio e longo prazo, aumentaria a confiabilidade nos serviços remotos.

Evidências têm mostrado que o uso da telessaúde pode trazer benefícios, como a redução de tempo de atendimento, dos custos de deslocamento de pacientes e profissionais de saúde e melhorias na qualidade assistencial, ao possibilitar o acesso a especialistas por profissionais de saúde não especializados de áreas remotas. (Caetano et al., 2020, p.3)

Entretanto, ainda que a telemedicina tenha alcançado algumas áreas isoladas, é de conhecimento geral que essa ainda possui limitações, uma vez que grande parte da sociedade brasileira sofre com as mazelas da desigualdade social, e, muitas vezes, não possuem aparelhos e nem redes de conexões qualificadas para se beneficiarem desse serviço, mostrando-se um desafio a ser enfrentado. “As dimensões do fenômeno de restrição de acesso aos serviços de saúde no âmbito socioeconômico envolvem as questões de oferta de equipamentos, distribuição de renda, saneamento básico, escolaridade, ocupação, gênero, raça e dimensão geográfica”. (Almeida, 2017, p. 4).

Com relação a telemedicina no contexto da COVID-19, o Gráfico 1 mostra que $92 \%$ percebe o crescimento dessa modalidade de atendimento durante a pandemia e que $7,7 \%$ concordam parcialmente. 
Gráfico 1. Você acredita que o contato com a telemedicina passou a crescer a partir da COVID-19?

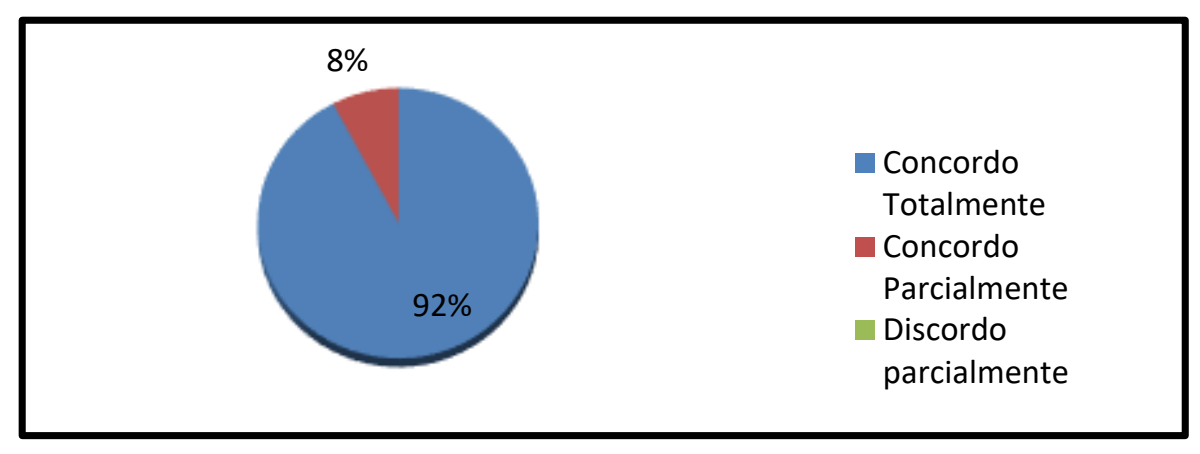

Fonte: Autores (2021).

Com a chegada do SARS-CoV-2 no ano de 2020, o SUS se mostrou bastante frágil, com isso foi necessário programar o uso de tecnologias integrativas com a finalidade de reduzir o contato social e garantir a segurança sanitária, evitando a propagação do vírus. A Telemedicina, nesse contexto, contribuiu com a assistência à saúde nas suas modalidades de teleassistência, teleconsulta, teletriagem e teleducação, além de possibilitar orientações, troca de saberes entre os profissionais, emissão de laudos.

A expansão durante o período da pandemia fez com que a Telemedicina se inserisse desde a Atenção Primária até os serviços de saúde especializados, principalmente no acompanhamento de pacientes com doenças crônicas, substituindo momentaneamente o atendimento presencial pelo virtual visando a manutenção do cuidado em saúde mesmo durante um período crítico, como: acompanhamento de pacientes portadores de Diabetes Mellitus, Insuficiência Cardíaca e Saúde Mental, que são áreas que demandam uma grande quantidade de tempo nos serviços de saúde, e, consequentemente, conseguiram liberar o fluxo para novos pacientes e desafogar os locais de atendimento, sendo importante, como afirma Sarmento (2019), habituar e preparar a população e os médicos para convier com essa forma da medicina.

Pensando ainda no contexto pandêmico, esse serviço pôde ser útil no controle de casos e em frear a disseminação do vírus, uma vez que tem sido e ainda é utilizado para rastreio e diagnóstico de pacientes com suspeita de contaminação pelo SARS- CoV-2, evitando, assim, o contato e contaminações desnecessárias, uma vez que por meio de conversas de vídeochamada seria possível orientar corretamente os pacientes, reduzir a sobrecarga das redes de atendimento e ajudar na contenção do número de EPI's, que por muitas vezes, mostraram-se escassos dentro desse período.

O cenário de pandemia levou o Congresso Nacional a aprovar a Lei n ${ }^{\circ} 13.989 / 20$, que autoriza o uso da telemedicina e da realização de consultas médicas à distância sem a necessidade de ter um profissional de saúde junto ao paciente, ou mesmo para uma consulta prévia, enquanto durar a pandemia. Assim, constatou-se que o estado pandêmico e a referida lei promoveram uma aceleração e antecipação nas discussões sobre telemedicina, o que oportunizou a sociedade brasileira a experiência do atendimento médico à distância.

Nesse contexto, é possível perceber que ainda que tenhamos leis que regulamentem esse serviço, boa parte das vezes elas são desconhecidas pela população, como é o exemplo estudado: $23,1 \%$ discordam totalmente e $15,4 \%$ discordam parcialmente, o que leva a uma desaprovação pela falta de conhecimento.

De acordo com Gráfico 2, percebeu-se que 42,3\% concordam totalmente que é possível manter uma rede de atendimento mista (Telemedicina + Atendimento Presencial) após a pandemia da COVID-19, já 53,8\% concordam parcialmente e 3,8\% discordam parcialmente. 
Gráfico 2. Você acredita que seja possível manter uma rede de atendimento mista (Telemedicina + Atendimento Presencial) após a pandemia da COVID- 19 ?.

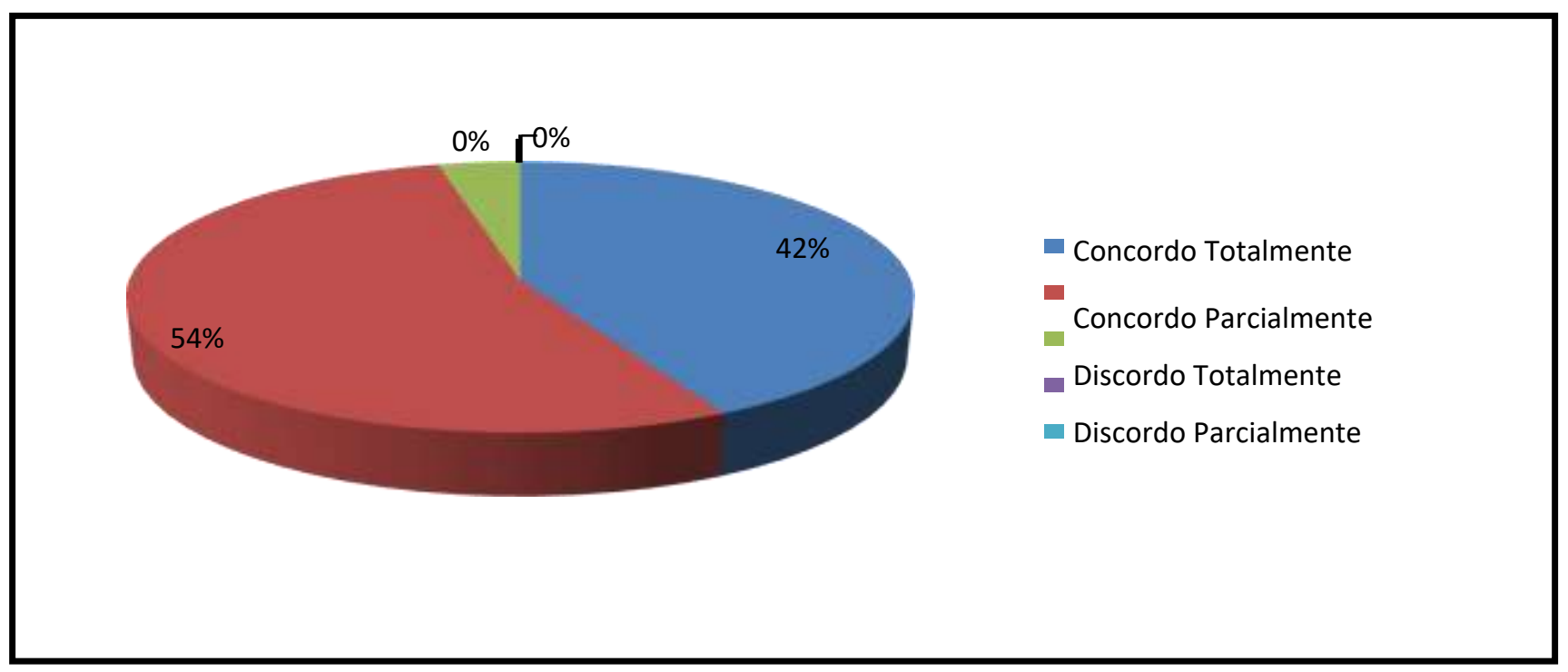

Fonte: Autores (2021).

Devido a pandemia do novo coronavírus, o estabelecimento do isolamento social fez surgir a necessidade de medidas para a desaceleração da transmissão do vírus, dessa forma, utilizando da telemedicina como meio de contenção e restrição de contato, como já mencionado. Mesmo em um contexto pós-pandemia, é possível que algumas dessas mudanças possam ser mantidas para garantir uma maior viabilidade do sistema de saúde, com a finalidade de torná-lo cada vez mais eficiente por meio do atendimento misto, tendo em vista que os avanços tecnológicos criaram novas formas de trabalho para os profissionais de saúde (Santos, 2020).

Além disso, a telessaúde ganhou uma atenção maior na utilização de tecnologias de informação, uma vez que hoje consegue auxiliar na realização de monitoramento, orientação e encaminhamento de pacientes a distância, bem como na troca de opiniões médicas e informações entre profissionais sobre os casos clínicos de alta complexidade.

Telemedicina está prevista pela Resolução CFM 1643/2002 (Conselho Federal de Medicina, 2002) e referendada pelo Ofício do CRM ao Ministério da Saúde (OFÍCIO CFM No 1756/2020 - COJUR) que dispõe o seguinte: Este Conselho Federal de Medicina (CFM) decidiu aperfeiçoar ao máximo a eficiência dos serviços médicos prestados e, EM CARÁTER DE EXCEPCIONALIDADE E ENQUANTO DURAR A BATALHA DE COMBATE AO CONTÁGIO DA COVID-19, reconhecer a possibilidade e a eticidade da utilização da telemedicina, além do disposto na Resolução CFM n ${ }^{\circ}$ 1.643, de 26 de agosto de 2002, nos estritos e seguintes termos: Teleorientação: para que profissionais da medicina realizam à distância a orientação e o encaminhamento de pacientes em isolamento; Telemonitoramento: ato realizado sob orientação e supervisão médica para monitoramento ou vigência à distância de parâmetros de saúde e/ou doença. Teleinterconsulta: exclusivamente para troca de informações e opiniões entre médicos, para auxílio diagnóstico ou terapêutico (Brasil, 2020, p. 26).

Dessa forma, o atendimento misto pode ser empregado de modo a manter o acompanhamento de pacientes crônicos e daqueles que já fazem acompanhamento regularmente nas unidades de saúde, como diabéticos, hipertensos e psiquiátricos, bem como as demais especialidades que consigam manter um atendimento de qualidade de forma online ou semipresencial, disponibilizando a demanda física para pacientes urgentes e que necessitem de um exame físico mais detalhado e profundo, garantindo, assim, uma maior agilidade no atendimento à toda população. Além disso, a telemedicina tem o potencial de promover uma maior integração do sistema de saúde (Maldonado, 2016; Ong, Pfeffer, \& Mullur, 2021). 
Analisando a questão número 21 "Quais estratégias, você como profissional da saúde acredita que possa ser eficiente para garantir um atendimento remoto ético e seguro?” Observou-se que as principais medidas, de acordo com as opiniões coletadas, para garantir um bom funcionamento da Telemedicina seriam: proporcionar plataformas onde ficariam salvos os atendimentos remotos, como uma espécie de prontuário eletrônico, atendimento híbrido, inserção da prática de telemedicina ainda na graduação e utilizar a telemedicina apenas para retorno, envio ou laudo de exames.

Em contrapartida, parte da amostra desacredita da segurança dos atendimentos remotos e afirmam que as consultas em telemedicina nunca serão substituídas pelo acompanhamento presencial, tendo em vista a complexidade da anamnese e do exame físico em algumas especialidades médicas. Dessa forma, foi realizada uma nuvem com as palavras chaves mais recorrentes nas respostas dos docentes foram: ética médica, telemedicina, acesso à saúde, segurança, tecnologia, atendimento, sigilo e capacitação como pode ser visualizado na Figura 1.

Figura 1. Nuvem de Palavras ilustrada a partir da pergunta 21 do questionário.

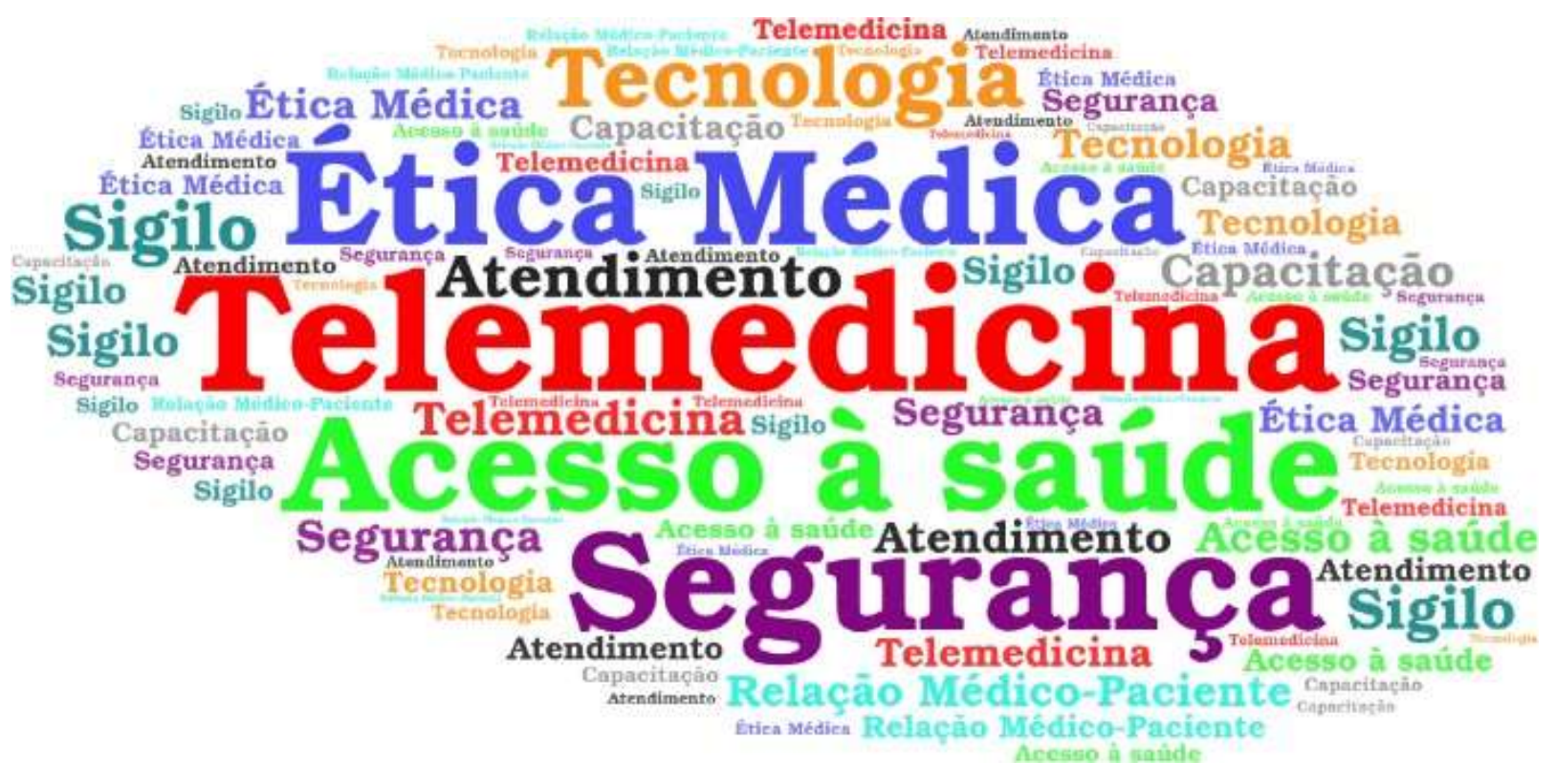

Fonte: Autores (2021).

\section{Conclusão}

As opiniões sobre a telemedicina ainda são divergentes. Parte dos profissionais da área médica ainda desacreditam ou se mostram receosos quanto a sua potencialidade e inserção no contexto da saúde, seja pela dificuldade do acesso integral ao paciente, pela complexidade envolvendo os aspectos éticos, ou pelo desconhecimento no que tange as leis que regulamentam esse serviço.

A telemedicina representa mais um avanço no contexto da saúde, é uma oportunidade de transformar a saúde num direito social, possibilitando o acesso a especialidades médicas com número reduzido de profissionais, bem como a ampliação das redes de saúde e garantindo maior cobertura ao paciente. Além disso, proporciona uma redução nos custos operacionais, que quando utilizado de forma estratégica pode estimular o reinvestimento em tecnologias, como exames de imagem, na infraestrutura dos locais de atendimento e ferramentas de segurança para proteção do paciente. (Lottenberg, 2021; Zanotto, 2020).

Vivemos em um país com elevado índice de desigualdade social, onde muitas pessoas ainda não possuem acesso a redes de computadores e internet de qualidade, dificultando o crescimento e consolidação da Telemedicina, e a garantia do 
acesso amplo e igualitário aos que necessitam desse serviço.

Dessa maneira, é possível que a Telemedicina sozinha ainda não consiga suprir todas as demandas de um atendimento convencional. Porém, com o surgimento do SARS-CoV-2 ela mostrou-se eficiente por meio das modalidades de atendimento misto, úteis para monitoramento de pacientes portadores de doenças crônicas e condições psiquiátricas. Tal conduta pode ser uma forma a ser utilizada para estruturação da telessaúde hoje, uma vez que consegue garantir uma maior flexibilidade de atendimentos e reduzir a sobrecarga do sistema de saúde.

Assim, entende-se que a visão dos docentes acerca da telemedicina é um problema multifacetário e sua discussão é de suma importância para o crescimento e aplicação dentro do mercado médico, considerando a adesão cada vez maior da população ao mundo digital, ainda que com limitações.

Diante da complexidade do tema e do baixo número de estudos científicos, sugere-se a continuidade das pesquisas para que os desafios envolvendo a Telemedicina possam ser superados, visando torná-la mais difundida, eficaz e adequada dentro do contexto médico.

\section{Referências}

Almeida, M. (2018). Desigualdade social e em saúde no Brasil: a telemedicina como instrumento de mitigação em João Pessoa-PB. Jornal Brasileiro de Economia da Saúde, 9(3), 292-303.

Amanda, O. C., Giovanna, C. B. L., Rosane, M. R., Yulle, R. M. \& Ana, C. P. (2018). Sistema de teletriagem para posto médico de saúde - sistema acolhe. In: XI simpósio de Engenharia biomédica, Uberlândia, MG.

Avila, R. (2003). Responsabilidades e Normas Éticas na Utilização da Telemedicina. Portal CFM.

Brasil (2020). Conselho Nacional de Secretarias Municipais de Saúde - CONASEMS. Guia orientador para o enfrentamento da pandemia Covid-19 na rede de atenção à saúde. Brasília.

Brito, B. \& Leitão, L. (2020). Telemedicina no Brasil: Uma estratégia possível para o cuidado em saúde em tempo de pandemia? Revista Saúde em Redes, 6(2), 7-19.

Caetano, R., Silva, A. B., Guedes, A. C. C. M., Paiva, C. C. N., Santos, D. L. \& Silva, R. M. (2020). Desafios e oportunidades para telessaúde em tempos da pandemia pela COVID-19: uma reflexão sobre os espaços e iniciativas no contexto brasileiro. Cadernos de Saúde Pública, 36(5), 1-16.

França, G. V. (2009). Telemedicina: breves considerações ético-legais. Revista bioética, 8(1), 107-126. https://revistabioetica.cfm.org.br/index.php/revista_bioetica/article/viewFile/266/266.

Khouri, S. (2003). Telemedicina: análise da sua evolução no Brasil. Dissertação (mestrado em ciências) Faculdade de Medicina da Universidade de São Paulo.

Lottenberg, C. (2021). Telemedicina é oportunidade real de transformar saúde em direito social. Global Summit Telemedicine \& Digital Health 2021. Disponível em: http://telemedicinesummit.com.br/artigo/telemedicina-e-oportunidade-real-de-transformar-saude-em-direito-social/.

Machado, F. S. N., Carvalho, M. A. P., Mataresi, A., Mendonça, E. T., Cardoso, L. M., Yogi, M. S., Rigato, H. M. \& Salazar, M. (2010). Utilização da telemedicina como estratégia de promoção de saúde em comunidades ribeirinhas da Amazônia: experiência de trabalho interdisciplinar, integrando as diretrizes do SUS. Ciência \& Saúde Coletiva, 15(1), 247-254.

Maldonado, J. M. S. V., Marques, A. B. \& Cruz, A. (2016). Telemedicine: challenges to dissemination in Brazil. Cadernos de Saúde Pública, 32(2).

Mariani, A. \& Pêgo-Fernandes, P.M. (2012). Telemedicina: Uma Revolução Tecnológica. Diagnostico e tratamento, 17(4), 155-156.

Oliveira, A. B., Tokarski, C. C. R., Japiassu, F. K. A. G. \& Silva, J. C. Q. (2020). Desafios do avanço da Telemedicina e seus aspectos éticos: revisão integrativa. Comunicação em Ciências da Saúde, 31(1), 55-63.

Ong, M. K., Pfeffer, M. \& Mullur, R. S. (2021). Telemedicine for adults. UpToDate. Disponível em: https://www.uptodate.com/.

Pereira, A. S., Shitsuka, D. M., Parreira, F. J., \& Shitsuka, R. Metodologia de pesquisa cientifica. UFSM

Resende, M. B., Carvalho, A. C. V., Fialho, B. B., Rezende, N. O. \& Moreira, M. E. C. (2019). Telemedicina e os impactos na assistência médica: uma revisão sistemática. Brazilian Journal of Surgery and Clinical Research, 28(2), 66-69.

Rezende, E., Melo, M. C. B., Tavares, E. C., Santos, A. F. \& Souza, C. (2010). Ética e telessaúde: reflexões para uma prática segura. Revista Panamericana de Salud Pública, 28(1).

Santos, W. S., Sousa Júnior, J. H., Soares, J. C. \& Rasch, M. (2020). Reflexões acerca do uso da telemedicina no Brasil: oportunidade ou ameaça? Revista de Gestão em Sistemas de Saúde, 9(3), 433-453. 
Research, Society and Development, v. 10, n. 14, e222101422224, 2021

(CC BY 4.0) | ISSN 2525-3409 | DOI: http://dx.doi.org/10.33448/rsd-v10i14.22224

Sarmento, I. P., Sarmento, R. P., Lopes, G. G. C., Bernardes, R. R., Netto, G. P. M \& Fontoura, H. S. (2019). Desafios da telemedicina no cenário brasileiro. Revista Educação em Saúde, 7(3), 42.

Zanotto, B. S., Etges, AP. B. S., Siqueira, A. C., Silva, R. S., Bastos, C., Araujo, A. L., Moreira, T. C., Matturro, L., Polanczyk, C. A. \& Gonçalves, M (2020). Avaliação Econômica de um Serviço de Telemedicina para ampliação da Atenção Primária à Saúde no Rio Grande do Sul: o microcusteio do Projeto TeleOftalmo. Ciência \& Saúde Coletiva, 25(4), 1349-1360. 